\title{
Microscopic Domain Structures in NiO Exchange-coupled Films
}

\author{
D. G. Hwang*, J. K. Kim¹, S. W. Kim¹, S. S. Lee, M. Dreyer ${ }^{2}$ and R. D. Gomez ${ }^{2}$ \\ Dept. of Computer and Electronic Physics, Sangji University, Wonju 220-702, Korea \\ 'Dept. of Physics Dankook University, Cheonan 330-714, Korea \\ ${ }^{2}$ Dept. of Electrical and Computer Engineering, University of Maryland, College park, MD 20742
}

(Received 2 August 2002)

\begin{abstract}
The dependence on nickel oxide thickness and a ferromagnetic layer thickness in unidirectional and isotropic exchange-coupled $\mathrm{NiO} / \mathrm{NiFe}(\mathrm{Fe})$ bilayer films was investigated by magnetic force microscopy to better understand the relation between magnetic domain structure and exchange biasing at microscopic length scales. As the NiO thickness increased, the domain structure of unidirectional biased films formed smaller and more complex in-plane domains. By contrast, for the isotropically coupled films, large domains generally formed with increasing $\mathrm{NiO}$ thickness including a cross type domain with out-of plane magnetization orientation. The density of the cross domain is proportional to exchange biasing field, and the fact that the domain mainly originated from the strongest exchange coupled region was confirmed by imaging in an applied external field during a magnetization cycle.
\end{abstract}

Key words : exchange biasing, MFM/STM, cross type domain

\section{Introduction}

Recently, the direct observations of magnetization reversal and domain structure in exchange coupled ferromagnetic (FM)/antiferromagnetic (AF) films have been conducted by several groups [1-7]. Most optical measurements reveal the macroscopic domain configurations and magnetization reversals of large sample areas above $100 \mu \mathrm{m}$ on a side. These observations revealed a distinct asymmetry of the FM domain wall motion and nucleation in decreasing and increasing magnetizing fields due to spiral spin rotation, acting as exchange spring [1], as well as the complicated FM structure due to non-uniform nature of exchange coupling $[3,4]$. While these macroscopic observations are valuable, it is hard to discern the behavior of microscopically inhomogeneous and often unstable exchange biasing (EB) of $\mathrm{NiO}$ film with grain size of $10^{1}$ $\mathrm{nm}$ or less. In the last few years, magnetic force microscopy was used to provide a more detailed view of complicated ripple domain. However, in MFM measurements by others on $\mathrm{NiO} / \mathrm{Co}$ bilayer [5] and patterned $\mathrm{NiO} / \mathrm{NiFe}$ elements $[6,7]$ the effects of locally randomoriented coupling were not addressed. In the former work,

*Corresponding author: Tel: +82-33-730-0413, e-mail: dghwang@ mail.sangji.ac.kr
Co, which has high coercivity $\left(\boldsymbol{H}_{C}\right)$ and small EB field $\left(\boldsymbol{H}_{e x}\right)$ was used; while in the latter, patterning created strong shape anisotropy induced domains. Thus, those experiments could mask the intrinsic effect of EB.

In this work, we studied continuous films of $\mathrm{NiO} /$ $\mathrm{NiFe}(\mathrm{Fe})$ bilayers by varying the thickness of the oxide film and the ferromagnet. Furthermore, the NiFe films were deposited with and without an external magnetic field $\left(\boldsymbol{H}_{d}\right)$, which produced unidirectional EB and isotropic EB films, respectively. Our motivation is that the microscopic domain structure of the isotropic-coupled $\mathrm{NiFe}$ layer may represent a more realistic picture for exchange coupling at the interface than the unidirectional biased NiFe layer. Among other things, we observed a cross type domain with magnetization of out-of plane in the isotropic exchange-coupled $\mathrm{NiO} / \mathrm{NiFe}(\mathrm{Fe})$ bilayer.

\section{Experimental}

$\mathrm{NiO}$ was prepared by if sputtering on $\mathrm{Si} / \mathrm{SiO}$ at room temperature without oxygen gas, and the NiFe film was deposited over the $\mathrm{NiO}$ without breaking vacuum, by $\mathrm{dc}$ sputtering with or without the $\boldsymbol{H}_{\boldsymbol{d}}$ of 300 Oe to get inplane unidirectional or isotropic EB. The structures are $\mathrm{Si} /$ $\mathrm{SiO} / \mathrm{NiO}(0,10,30,60 \mathrm{~nm}) / \mathrm{NiFe}(10 \mathrm{~nm})$. The magnetic properties were characterized from anisotropic magneto- 
resistance curves using 4-point terminal method. Topological and magnetic structures of NiFe film were subsequently measured by tapping mode AFM and MFM. The images of thin Fe films grown on polycrystalline $\mathrm{NiO}$ are measured in-situ by UHV STM/MFM measurements.

\section{Results and Discussions}

The $\boldsymbol{H}_{\boldsymbol{x}}$ of the unidirectional bilayer was almost zero for $10 \mathrm{~nm} \mathrm{NiO}$, and increased to $75 \mathrm{Oe}$ at $60 \mathrm{~nm} \mathrm{NiO}$. In general, a thin $\mathrm{NiO}$ layer below a critical thickness of about $30 \mathrm{~nm}$ contains unstable $\mathrm{AF}$ grains and weak $\mathrm{EB}$ with FM layer because of its low magnetocrystalline anisotropy. With the thin AF layer, as the magnetization of FM layer is rotated, the weak AF grains themselves can become unstable and switch. On the other hand, as the thickness increases, $\mathrm{NiO}$ film grows in fine columnar grain with a small diameter below $30 \mathrm{~nm}[3,5]$, and stable AF grains strongly couple with FM layer. The FM layer receives a locally different $\boldsymbol{H}_{\boldsymbol{e x}}$ from a randomly oriented AF grains, and the magnetization reversal takes place at different external fields for different FM domains.

The aforementioned statements are elucidated by the MFM images of the NiFe domain structures of the isotropic and unidirectional exchange-coupled bilayers as functions of NiO thickness. Figures 1(a)-(d) show the evolution of the microscopic ripple domain pattern of the unidirectional bilayer from the mesh type pattern with no $\mathrm{NiO}$ to a more complicated and coarse-grained structure as $\mathrm{NiO}$ increased to $60 \mathrm{~nm}$. The mesh type ripples of $\mathrm{EB}$ NiFe films are the well-known to be a combination of longitudinal and transverse ripples due to small variations in local magnetization [8]. The ripples are easily removed at an applied field of 3 Oe. But the complicated ripple pattern of the $60 \mathrm{~nm} \mathrm{NiO}$ bilayer persists even up to 100 Oe, with the pattern preserved until magnetization direction was reversed. These small and strongly pinned ripples almost certainly originate from the interface coupling of randomly oriented AF grains. However, it is difficult to distinguish the ripple structures between the bilayers having a different $\boldsymbol{H}_{e x}$, because ones deposited with $\boldsymbol{H}_{\boldsymbol{d}}$ were forced to have strong unidirectional inplane magnetization by the magnetic field. For example, we established that the $\boldsymbol{H}_{\boldsymbol{e}}$ of the unidirectional biased $\mathrm{NiO}(60 \mathrm{~nm}) / \mathrm{NiFe}(10 \mathrm{~nm})$ bilayer is strongly dependent upon $\mathrm{NiO}$ deposition rate. It varied from 75 Oe for $3 \AA /$ min to 103 Oe for $12 \AA / \mathrm{min}$. However, the ripple structures we observed were identical.

In order to study a more realistic representation of the local coupling distribution, the MFM images of the isotropic coupled bilayers were obtained. Some representative results are shown in figures $1(\mathrm{e}) \sim(\mathrm{h})$. We note that as the $\mathrm{NiO}$ thickness was increased, the magnetization direction rotated from in-plane to out-of-plane. Even at $10 \mathrm{~nm} \mathrm{NiO}$, the mesh type ripples have all but disappeared. At $30 \mathrm{~nm}$ $\mathrm{NiO}$ thickness, high contrast and large domains appear in stark contrast with the unidirectional films. Upon closer inspection, we found that the magnetization patterns contained unusual domains, which we refer to as "crosstype domains". These are most evident in the $60 \mathrm{~nm} \mathrm{NiO}$ bilayer as shown in the marked circles of figure 1(h). A high-resolution image of the cross-type domain is shown in figure 2 where we rescanned a section of figure 1(h) amplified $4 \mathrm{x}$ and 10x. The bright areas and dark areas that comprise the horizontal and vertical legs of the cross are from the magnetic poles of the out-of-plane magnetization. The domains became smaller but had stronger contrast as NiFe thickness was decreased. We investigated the difference in cross domain structure as a function of deposition rate and hence, $\boldsymbol{H}_{\boldsymbol{e x}}$, and found that the density of these cross-type is proportional to the value of the $\boldsymbol{H}_{e x}$. Thus, we believe that these domains are responsible for $\boldsymbol{H}_{\text {ex }}$.

To explain the origin and structure of the cross-type domain a schematic of the interfacial cross section of the unidirectional and isotropic coupled FM layer over an

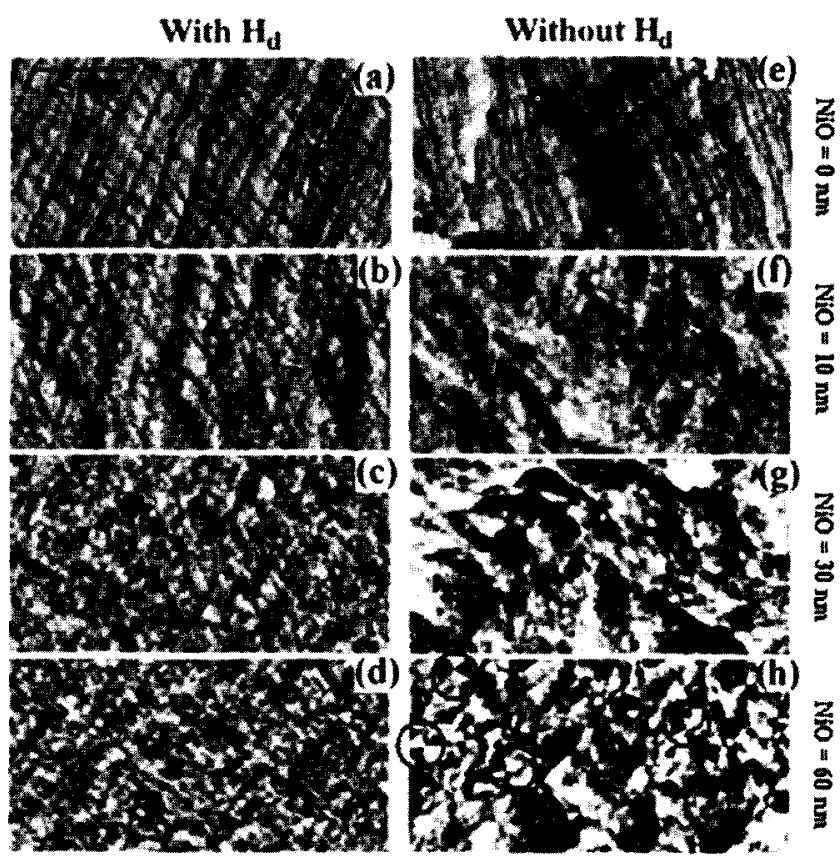

Fig. 1. The dependences of microscopic MFM images on $\mathrm{NiO}$ thickness in the $\mathrm{NiO}(\mathrm{t} \mathrm{nm}) / \mathrm{NiFe}(10 \mathrm{~nm})$ bilayer deposited with and without $\mathbf{H}_{\mathbf{d}}$, where $t$ is (a, e) $0 \mathrm{~nm},(\mathrm{~b}, \mathrm{f}) 10 \mathrm{~nm},(\mathrm{c}, \mathrm{g}) 30$ $\mathrm{nm}$, and (d, h) $60 \mathrm{~nm}$. The (a) (d) images were measured from the bilayers with $\mathbf{H}_{\mathbf{d}}$ and the $(\mathrm{e}) \sim(\mathrm{h})$ ones without $\mathbf{H}_{\mathbf{d}}$. The marked circles show the cross-type domains. 


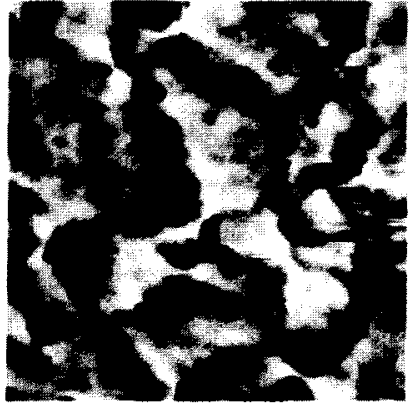

(a) $5 \times 5 \mu \mathrm{m}$

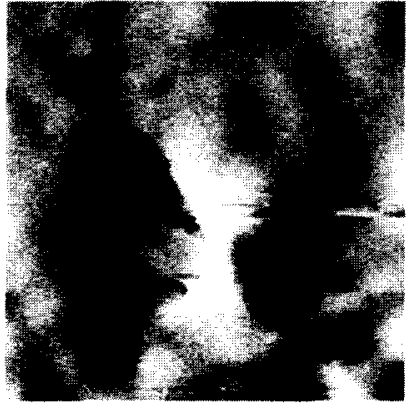

(b) $2 \times 2 \mu \mathrm{m}$
Fig. 2. The (a) $5 \times 5 \mu \mathrm{m}$ and (b) $2 \times 2 \mu \mathrm{m}$ scanned high-resolution image of cross-type domain in the $\mathrm{NiO}(60 \mathrm{~nm}) / \mathrm{NiFe}(10$ $\mathrm{nm}$ ) bilayer without $\mathbf{H}_{\mathbf{d}}$

(111) oriented and tilted AF grain are suggested in figure 3. The schematic is based on high-resolution TEM micrographs of $\mathrm{NiO} / \mathrm{Co}$ interfaces measured by others [3]. The $\mathrm{NiFe}$ film deposited without $\boldsymbol{H}_{\boldsymbol{d}}$ over randomly oriented $\mathrm{NiO}$ grains preferentially contain a higher density of outof-plane moments in comparison with those deposited with an in-plane $\boldsymbol{H}_{\boldsymbol{d}}$ field. The in-plane $\boldsymbol{H}_{\boldsymbol{d}}$ field forces the successive atomic layers to orient parallel to the surface, so that the number of vertical moments decreases with increasing thickness. As a consequence, the moments near at the surface of the $10 \mathrm{~nm}$ thick NiFe films are preferentially in-plane and lead to the MFM image in figure 1(a) (d). The complex ripple pattern observed for the exchanged biased films arise from the local variation of the in-plane magnetization, $\operatorname{Div}^{*} \mathrm{M}$, possibly reflecting the grains of the underlying $\mathrm{NiO}$ films. A similar explanation is presumably valid for the case when the grown films are magnetically annealed. For the case isotropic films, the aligning field is absent during growth so that the ferromagnetic exchange interaction forces the $\mathrm{NiFe}$ film to follow the underlying moment of the EB layer which consequently lead to a large out-of-plane component. Despite the fact that the $\mathrm{NiO}$ grains are on the order of $10^{1} \mathrm{~nm}$, the strong ferromagnetic coupling between $\mathrm{NiFe}$ causes the formation of domains with out-of-plane magnetization. These domains are most likely circular by symmetry considerations, and the center is quite possibly pinned by regions of locally strong exchange coupling with the $\mathrm{NiO}$. As the $\mathrm{NiFe}$ thickness increases the size of the domains similarly increase as observed in our experiments. These domains possess high magnetostatic energy which is then reduced by the break-up of domains in the cross-type configuration as shown in the sketch in figure 3(c). These cross-type domains are quite different from other systems that have out-of-plane magnetizations such those seen in multilayer films used in perpendicular
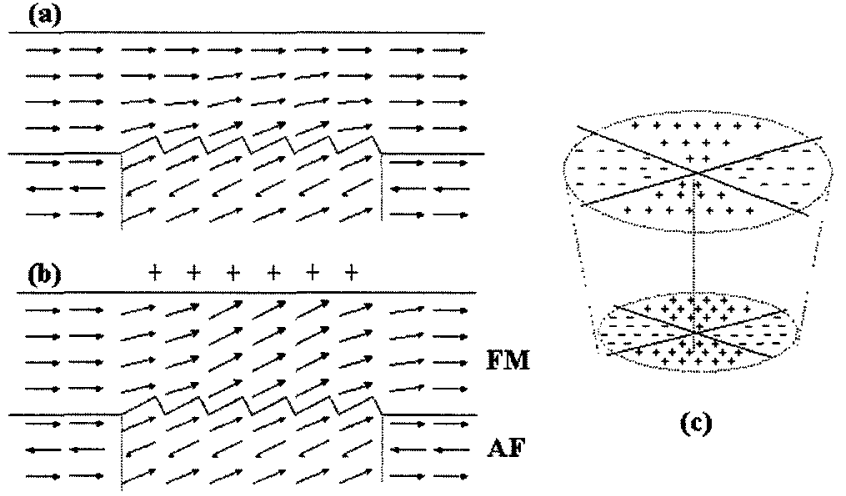

(c)

Fig. 3. A schematic views of the interfacial cross sections of an (a) unidirectional and (b) isotropic exchange-coupled FM layer over a (111)-oriented and tilted AF grain. Each arrow represents average magnetization vector of several spins. (c) A schematic view of a typical cross-type domain with out-of magnetic poles

recording. In those systems, one invariably finds laryrinth or serpentine patterns that are expected to form in the absence of underlying pinning centers afforded by the ferromagnetic exchange coupling.

Finally, an alternative explanation for the observed effect is as follows. The observed grain sizes of polycrystalline $\mathrm{NiO}$ film were reported to be a few ten nanometers $[3,5]$ but the sizes of these domains were about $1 \sim 2 \mu \mathrm{m}$ in $\mathrm{NiFe}(10 \mathrm{~nm})$. Although the domain size was reduced with decreasing $\mathrm{NiFe}$ thickness, it could not explain that each domain has originated from one tilted $\mathrm{NiO}$ grain. To settle this apparent inconsistency, our model can be supplemented from Takano's model for the uncompensated interfacial AF spins [9]. The cross domain can be regarded as caused by the uncompensated poles of several $\mathrm{NiO}$ grains rather than one tilted grain. Also, even if the local out-of-plane pole was from each tilted (111) grain, the macroscopically observed pole density will be reconstructed by a strong exchange coupling between FM spins. Conclusively, one can argue that the preponderance of the cross-type domains reflect a high density of uncompensated moments at FM/AF interface; and these uncompensated moments generate a strong exchange biasing.

Figure 4 shows a possible process on the creation and field induced variations of cross-type domain in $\mathrm{NiO}(60$ $\mathrm{nm}, 3 \AA / \mathrm{min}) / \mathrm{NiFe}(10 \mathrm{~nm})$ bilayer during a magnetization cycle. As the external field is released from positive saturation, a strong contrast out-of-plane pole was created at zero field as shown in figure $4(\mathrm{c})$. Note that the scanned image (c) is different from (d) despite being obtained in a same region and field. Prior to the creation of the cross-type domain at (d), a single out-of-plane 


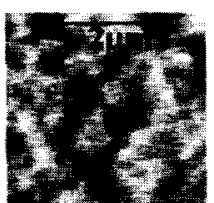

(a) 2100

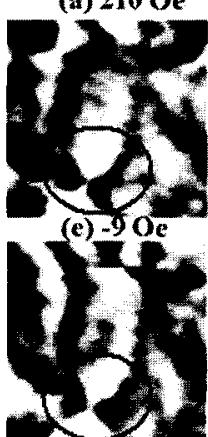

(i) $90 \mathrm{e}$

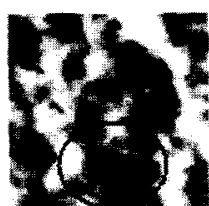

(b) $30 \mathrm{Oe}$

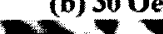

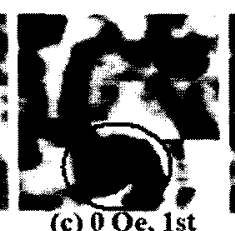

(c) 0 Oe, ist

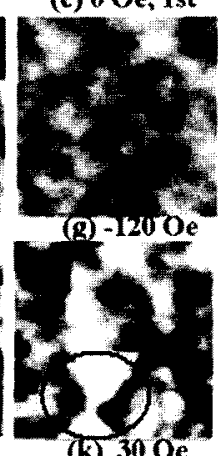

(k) $300 \mathrm{e}$

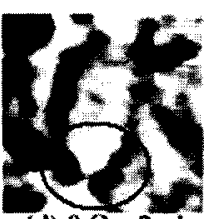

(d) 00 e, ind

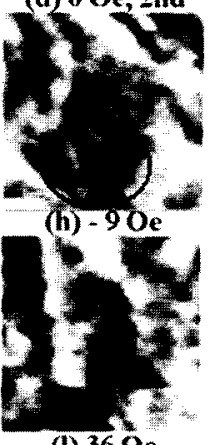

(1) 360
Fig. 4. MFM images of cross type domain changing as applied field decreases (a) to $(\mathrm{g})$, and increases $(\mathrm{g})$ to $(\mathrm{k})$. The cross domain is at the marked circles, and the scratched lines in the images of (c), (f), and (k) were influenced by MFM tip.

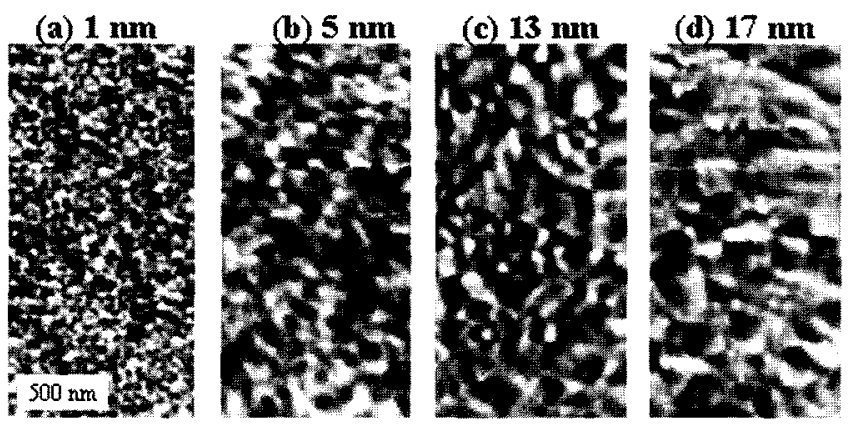

Fig. 5. MFM images of exchange-coupled NiO/Fe films as Fe thickness increase (a) $1 \mathrm{~nm}$ to (d) $17 \mathrm{~nm}$.

domain was concentrated at the same region. The field from the MFM tip provided that additional perturbation to induce the transformation into a cross-type domain which reduces the pole energy. More importantly, we observed that despite difference elsewhere in the domain configurations with increasing and decreasing fields, the cross type domain invariably appeared at the same region and with nearly identical structure. It is strong evidence that the domain was induced from the strongest exchange coupling of FM/AM interface.

Figure 5 shows the MFM images of thin Fe films grown on polycrystalline $\mathrm{NiO}$ using in-situ UHV MFM/
STM measurements. As the Fe thickness increases $1 \mathrm{~nm}$ to $17 \mathrm{~nm}$, the magnetic domain structure changed from the fine complex structure of $1 \mathrm{~nm}$ Fe film to the cross type domain structure above $10 \mathrm{~nm}$ Fe.

\section{Conclusion}

We observed a new cross-type domain with strong out-of poles in isotropic exchange-coupled $\mathrm{NiO} / \mathrm{NiFe}$ film. From the data on the dependence on thickness and deposition rate, the existence of the domain has been directly linked with the local exchange biasing field. The fact that the domain originated from the strongest exchange-coupling region was confirmed by dynamic magnetic domain configurations during a magnetization cycle and the MFM images of different Fe thickness.

\section{Acknowedgements}

This work was supported in part by the Korea Research Foundation under Grant No. 2000-013-EA0125 and Sangii University.

\section{References}

[1] V. I. Nikitenko, V. S. Gornakov, A. J. Shapiro, R. D. Shull, K. Liu, S. M. Zhou, and C. L. Chien, Phys. Rev. Lett. 84, 765 (2000); Phys. Rev. B 57, R8111 (1998); J. Appl. Phys. 83, 6828 (1998).

[2] X. Portier, A. K. Petford-Long, S. Mao, A. M. Goodman, H. Laidly, and K. O'Grady, IEEE Trans. Magn. 35, 3091 (1999).

[3] H. D. Chopra, D. X. Yang, P. J. Chen, H. J. Brown, L. J. Swartzendruber, and W. F. Egelhoff, Jr, Phys. Rev. B 61, 15312 (2000); J. Appl. Phys. 87, 4942 (2000).

[4] Z. Qian, M. T. Kief, P. K. George, J. M. Sivertsen, and J. H. Judy, J. Appl. Phys. 85, 5525 (1999).

[5] M. Cartier, S. Auffret, Y. Samson, P. Bayle-Guillemaud, and B. Dieny, J. Magn. Magn. Mater. 223, 63 (2001).

[6] J. Yu, A. D. Kent, and S. S. Parkin, J. Appl. Phys. 87, 5049 (2000).

[7] J. Ding and J. Zhu, J. Appl. Phys. 79, 5892 (1996); J. C. Wu, H. W. Huang, C. H. Lai, and T. H. Wu, J. Appl. Phys. 87, 4948 (2000).

[8] M. Prutton, Thin ferromagnetic films, Butterworth, Washington D.C., 1964, pp. 162.

[9] A. E. Berkowitz and K. Takano, J. Magn. Magn. Mater 200, 552 (1999); Phys. Rev. Lett. 70, 1130 (1997). 